\title{
Supermedia in Internet-Based Telerobotic Operations ${ }^{\star}$
}

Imad Elhajj ${ }^{1}$, Ning Xi ${ }^{1}$, Wai Keung Fung ${ }^{2}$, Yun hui $\mathrm{Liu}^{2}$, Wen $\mathrm{J.} \mathrm{Li}^{2}$, Tomoyuki Kaga ${ }^{3}$, and Toshio Fukuda ${ }^{3}$

1 Dept. of Electrical and Computer Engineering, Michigan State University, East Lansing, MI 48824, U.S.A.

2 Dept. of Automation and Computer-Aided Engineering, Chinese University of Hong Kong, Shatin; N.T., Hong Kong

3 Center for Cooperative Research in Advanced Science and Technology, Nagoya University, Nagoya 464-8603, Japan

\begin{abstract}
In the past decade robotics and the Internet, fed by the great advances in computing and networking, matured into giant interdisciplinary scientific fields. Therefore, it is not surprising that many are trying to merge these two technologies and develop Internet-based robotic teleoperation. More interestingly, Internet-based bilateral teleoperation, where supermedia is fed back to the operator in order to increase efficiency and achieve telepresence. Supermedia is the collection of multimedia (video, audio, ...), haptic and other sensory information. This paper studies supermedia enhanced teleoperation via the Internet, concentrating on the real-time control of such systems and their performance. The potential of this technology and its advantages will be explored. In addition, key issues, such as stability, synchronization and transparency, will be analyzed and studied. Specifically, event-based planning and control of Internet-based teleoperation systems is presented with experimental results of several implemented system scenarios.
\end{abstract}

\section{Introduction}

When it comes to robotics, the lack of applications has never been an issue. The same can be said for Internet-based teleoperation, where the potential for applications is just limited by our imaginations. One of those applications is telemedicine, where several successful experiments have been done in this field. The main use of tele-medicine is in remote checkup and surgery, home care (elderly and disabled remote assistance) and physical therapy [1] [2]. Another application is operations in hazard environments, most commonly hazard material manipulation [3], space and deep sea operations [4] [5]. In addition e-services, such as remote maintenance and monitoring of manufacturing processes, equipment and products. Also the ability to use sophisticated and expensive manufacturing facilities by several users around the world is very cost effective [6]. Other

\footnotetext{
* Research Partially supported under NSF Grant IIS-9796300 and IIS-9796287.
} 
applications relate to law enforcement. Government agencies are already using remotely operated machines in their operations to reduce human risk. Also Internet-based teleoperation can be applied to entertainment. There are already on the web teleoperated games, where users can remotely login and operate a robot. This application will experience great advance in the coming few years once businesses realize its potential.

However, before these applications become widely accepted and used, several things have to be supplied and ensured. Most importantly is telepresence, which is the transfer of human senses to remote locations by feeding back sensory information from the remote environment. This would closely couple the operator with the remote environment and thus gives a more realistic feeling of remote presence. In addition to realism, telepresence significantly increases the efficiency of teleoperation. To achieve this, sensory information has to be presented to the operator, the most common of which is visual feedback. However this is not sufficient for some applications; therefore, additional types of feedback are required. Supermedia feedback, which can correspond to different sensory information, considerably increases operators' efficiency and makes some tasks feasible [7] 8]. The general structure of such a system is shown in Fig 1] where the operator sends velocity commands and receives supermedia (visual and haptic) information.

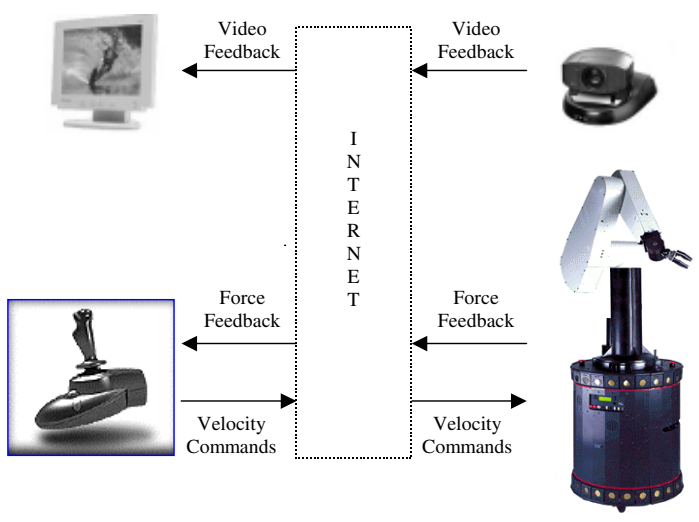

Fig. 1. General structure of an Internet-based teleoperation system with supermedia feedback.

Examining the literature, Internet-based robots can be divided into three categories according to the method used to send commands and receive haptic feedback and according to the nature of feedback supplied. These three categories are referred to here as: teleprogrammed, telesimulated, real real-time teleoperated.

Teleprogrammed Internet-based robots are ones that require the operator to upload a plan or set of commands for it to execute. This uploaded program is 
executed by the robot either autonomously or semi-autonomously. This category can be further divided into "program and watch" robots or "program, watch and intervene" robots. Typically, teleprogrammed robotic systems include only visual feedback [9]-11.

Telesimulated Internet-based robots include systems that feed forward commands in real-time but the feedback in simulated. This simulated or predicted feedback can be one of two forms; either it is completely simulated based on a robot and environment model, or it is partially simulated based on a model and corrected by actual feedback. Generally feedback is in the form of predicted display and/or predicted force [12] 13.

Real real-time teleoperated Internet-based robotic systems feed forward commands in real-time and feedback real real-time sensory information. The feedback comes in several forms the most typical of which are video and force [14] 15.

As in any control system, in order for Internet-based bilateral control to be acceptable it has to satisfy several performance criteria. Stability is the main one since it relates to feasibility and safety of the operation. The other two are, synchronization and transparency, which relate to the efficiency of the operation and its realism.

However, when it comes to the Internet, ensuring those performance characteristics becomes a significant challenge. The random time delay, network buffering effects and disconnections experienced over the Internet present major difficulties. Those difficulties have been extensively studied in the literature, especially resolving time delay effects in teleoperation systems with haptic feedback [7] [16]-19]. But all those studies have several limitations since they assume time delay to be either fixed, the same in both directions or has an upper bound, none of which applies to Internet type delays. So there is a need for a planning and control method that can achieve stability, synchronization and transparency of Internet-based teleoperation with real-time supermedia feedback regardless of time delay and its variance. This paper presents such a method, as well as theoretical and experimental results.

\section{Supermedia in Closed-Loop Control of Internet-Based Teleoperation}

Initially most teleoperation done was open-loop, meaning the only form of feedback was video. Once haptic feedback started being considered, the control loop was closed between the robot and operator. Haptic information is that relating to the sense of touch, in other words tactile information. This information can be fed back, where it is reproduced to the operator using force generating devices. The haptic information does not always correspond to actually physical forces but can also correspond to other sensory information, such as heat, radiation and distance to obstacles. The need for haptic feedback is to increase the efficiency of teleoperations. However, it does not substitute the need for visual feedback, which is still an important form of feedback. Actually any type of multimedia (audio, video ...) can be fed back to increase efficiency and telepresence. 
To capture the notion of all these feedback streams the term supermedia is presented. Supermedia is the collection of multimedia, haptic and any other sensory information. So the interest of this paper is in studying the effect of varying time delay on Internet-based teleoperation systems that include supermedia. And to develop a new planning and control methodology that would ensure stability, synchronization and transparency regardless of the time delay faced. This time delay, where in the Internet can not be predicted and is random with a very complex stochastic model.

Several attempts have been made to model delay over the Internet, and several complex models have been derived [20]. In 20] a wavelet-based model was developed, where delay was shown to be self-similar 21 in a statistical sense. However, estimating the arrival time of packets is still difficult to achieve. Fig.2 gives an example of the round trip time (RTT) between Michigan State University and Hong Kong, where the randomness of RTT is clearly shown.

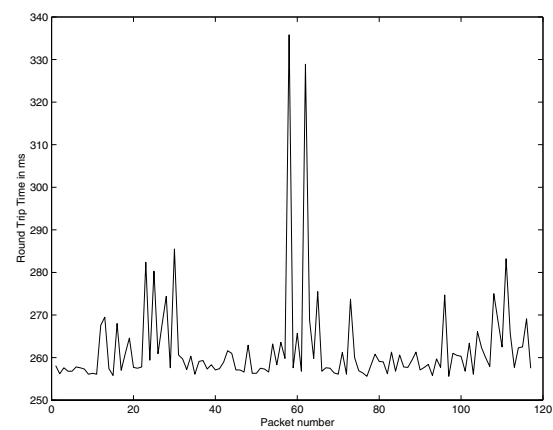

Fig. 2. Round trip delay in ms for packets transmitted between the robot and Hong Kong station.

This interest placed on time delay is a result of the instabilities it causes. Since delays on the order of a tenth of a second can destabilize the teleoperator [7]. And when haptic feedback is being used the system is stabilized only when the bandwidth was severely reduced. In addition to instability, de-synchronization can occur between the different supermedia streams. Desynchronization is a result of the buffering effect delay creates, where the network behaves as a FIFO buffer. This results in desynchronization between the feedback and feed forward streams thus destabilizing the system, and between the feed back streams themselves thus resulting in operator confusion and efficiency reduction. As for transparency, delay causes high frequency haptic changes to be filtered thus reduces the transparency of the operation. 


\section{Event-Based Planning and Control of Internet-Based Teleoperation}

The performance effects discussed in the previous section are a result of using time as a reference for the different system entities. So intuitively if a different non-time based reference can be used these effects can be eliminated or at least reduced significantly. In traditional control systems the dynamics of the system is modeled by differential equations in which the free variable is the time variable $t$. And usually the trajectory is a function of time, but if we do not constraint the trajectory by time we would allow the system to be at any point at any time. So the general idea is to model the system and the trajectory with parametric equations. The parameter is called motion reference or action reference, and usually denoted by $s[22$.

This planning and control method is referred to as event-based, which was extended to bilateral teleoperation applications in 23]. This extension had to make some alterations to the initial event-based theory in order to be fit for teleoperation applications. The first main thing that had to be considered was the fact that in teleoperation there is no predefined path. The path is generated in real-time by the operator based on the feedback received. The other issue is that the operator has limited computational power so a simple event or reference has to be chosen. In addition, the method had to be independent of the operator and environment model, and it should not require significant overhead.

Therefore, the event, $s$, had to be carefully chosen in a way that no overhead is required from the operator and it had to be intuitive. In addition, to maintain the synchronization between the operator and the remote machine regardless of time delay the event had to be designed in a way to eliminate the buffering effect of delay. This effect is shown in time based part of Fig. 3 where it is clear in the time based approach that the delay will cause many signals to be flowing within the network. So by the time a new command, $V_{t+m}$, that was generated as a result of a force, $F_{t+k}$, arrives at the destination, the state of the machine would have changed due to the commands that were already buffered in the network, $V_{t+1}, V_{t+2}, \ldots V_{t+m-1}$. This implies that when the robot gets the command $V_{t+m}$ at time $t+m+n$, it has already changed status and is being subject to the force $F_{t+n+m}$; therefore, the command would be a wrong one since it was a result of $F_{t+k}$ and not $F_{t+n+m}$.

In order to ensure that the right command is being received and executed the buffering effect should be eliminated. So the communication and control model should be similar to the one shown in the event based part of Fig 3 As shown, the transmission of new commands and forces is not specified by time. A new command, $V_{n+1}$, will not be generated and sent until the most up-to-date status of the robot, $F_{n}$, is received. At the same time, a new force, $F_{n+1}$, will not be fed back until a new velocity, $V_{n+1}$, is received. This implies that there are no commands or forces within the network flowing thus the buffering effect is eliminated.

This setup is achieved by using event-based planning and control, with the event taken as the command number. So if currently the operator is sending the 

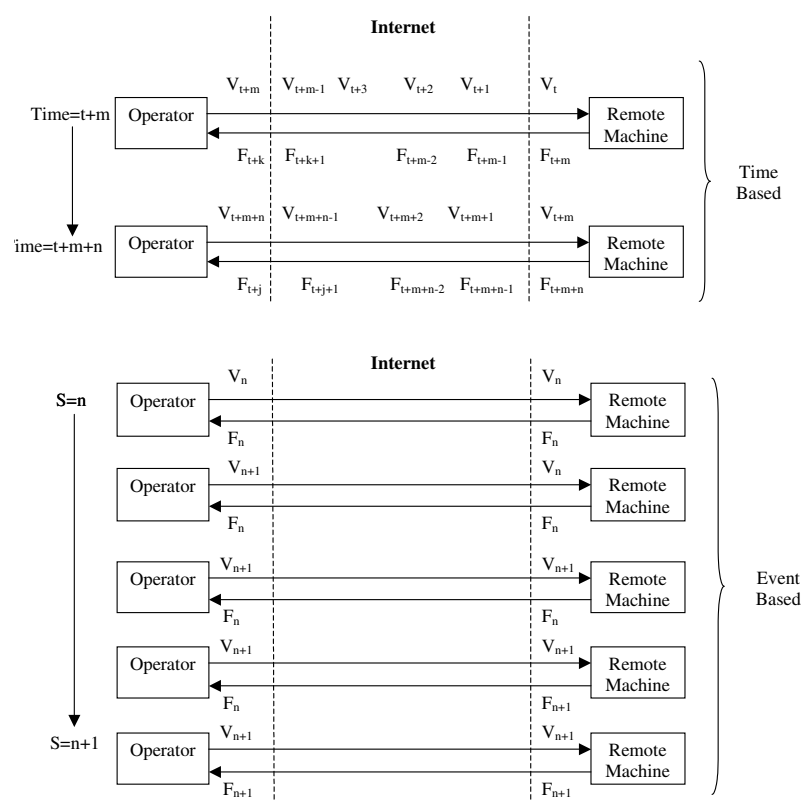

Fig. 3. Illustration of the buffering effect of delay, and the elimination of this effect in event-based planning and control.

$n^{t h}$ command, then the event is $n$. This choice of the event is intuitive and does not require the operator to keep track of it, since the communication procedure will take care of ensuring this specific behavior of the system regardless of the operator's command. This is an additional advantage to this approach since the whole procedure mentioned is transparent to the operator.

\section{Performance Analysis of Internet-Based Teleoperation}

This section will analyze the stability, synchronization and transparency of the event-based approach. Concerning the stability of the system under event-based referencing, the following theorem was proven in [22]:

Theorem 1. If the original robot dynamic system (without remote human / autonomous controller) is asymptotically stable with time $t$ as its action reference; and the new non-time action reference, $s=\prod(y)$ is a (monotone increasing) nondecreasing function of time $t$, then the system is (asymptotically) stable with respect to the new action reference $s$.

The only assumption needed is that the robot is a stable system, which means that the original robot dynamic system (without remote human operator) is asymptotically stable with $\mathrm{t}$ as its action reference. This would allow the use of Theorem 1 and proves the (asymptotical) stability of the system with respect 
to the new action reference $s$, simply by proving that the new non-time action reference is (monotone increasing) non-decreasing function of time $t$. The advantage of this approach is that stability is proven independent of the human model or the statistics of time-delay. Clearly the choice of event used in this approach gives an event that is a non-decreasing function of time, since the command numbering can only increase. Therefore, the event-based planning and control for teleoperation with haptic feedback results in a stable system despite random time delay.

Examining the event-based case in Fig, 3 it is clear that the update of signals in both directions is not triggered by time. Also since the buffering effect of delay is eliminated then the haptic force felt by the operator is the most up-to-date one; there could not have been any change in the system status meanwhile because there are no velocity commands flowing in the network. The same thing applies to the velocity received by the robot, it is the most up-to-date one since there were no new forces flowing in the network that could have generated new commands. This implies that the operator and the robot are always synchronized in event regardless of time delay and its variation. These two different entities in the system can not be at different events; in a closed loop system, the feedback obtained has to correspond to the most up-to-date status of the plant being controlled.

From the analysis in the previous section it is clear that the frequency of events is a function of time delay. Since we have an event per round trip delay then the frequency by which the haptic information is fed back is a function of time delay. This implies that the force will be sampled once for each round trip delay and since this delay is variable then the sampling of force is variable. From Shannon's sampling theorem we know that in order to reconstruct a signal perfectly from a sampled one, the sampling rate should at least be twice $F_{\max }$, where $F_{\max }$ is the highest frequency component of the original signal. So as long as the round trip delay is less than the inverse of the highest frequency component of the force applied on the robot, then the fed back haptic force can be used to regenerate the actual one almost perfectly and thus the system transparency is achieved. Once the time delay increases beyond that value the transparency will decrease accordingly. So this approach does not ensure perfect transparency regardless of time, but it does ensure that the best transparency will be achieved for a certain time delay.

\section{Experimental Implementation and Results}

The details of several experimental setups will be given with their results. These results will confirm the analysis done in the previous section. The first scenario is the teleoperation of a mobile robot with haptic feedback. The feedback in this case corresponds to the distance to obstacles detected in the environment. So the operator is able to sense the obstacles in front of the robot before hitting them. The general model of such a system is shown in Fig 4 . 


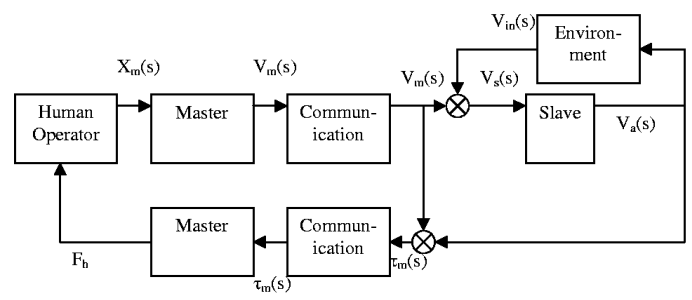

Fig. 4. The system architecture of the mobile base teleoperation system.

This model shows that the operator is sending velocity commands and receiving haptic information corresponding to sensory information from the environment. This system was experimented with an operator in Hong Kong controlling the robot at Michigan State University in real-time. The results in Fig 5 are showing the plot of time versus $s$, the event, in the first row. The other plot in the first row shows the desired rotational velocity. The second row shows the desired velocities in $x$ and $y$ directions, which is called $V_{m}$. The third row displays the actual velocities in both directions, which is called $V_{a}$. Next we illustrate the force that is played by the joystick in both directions, called $\tau_{m}$. The last row displays the same plot, which is the closest detected distance to obstacles in the environment.

As seen, the actual velocities are tracking the desired ones as long as the distance to obstacles is less than a critical distance. Therefore, the system output stability is illustrated. In addition, the change of the desired and actual velocity direction is occurring at the same event, thus the system is synchronized.

The second system implemented was a mobile manipulator that is controlled in real-time, where the operator sends a velocity command that is divided by the local controller between the arm and the mobile base. This division is done to achieve an acceptable posture for the arm, so if the robot arm is almost fully extended or extracted, the mobile platform will be repositioned to achieve a better posture. The haptic feedback in this case is actual force detected by the force/torque sensor mounted on the gripper.

The experimental procedure was to have the operator in Hong Kong move the robot in $x$ and $y$ directions randomly. Meanwhile, a person will subject the robot gripper to forces in the $x$ and $y$ directions randomly. The average frequency of communication was $3.1 \mathrm{~Hz}$, which implies that on average we had 3.1events/sec.

The main interest was in how close the actual velocity of the robot tip was following the desired velocity specified by the operator, and in how close was the force felt by the operator close to the one detected by the force/torque sensor. In other words, how were the operator's intentions executed by the mobile manipulator and how the environment was felt by the operator, despite time delay and time delay variance.

The results are shown in Fig, 6 and Fig[7. In Fig [6 the first row gives the operator's desired velocity in the $x$ and $y$ directions with respect to time. The second row is the actual velocity of the arm tip in the $x$ and $y$ directions with 

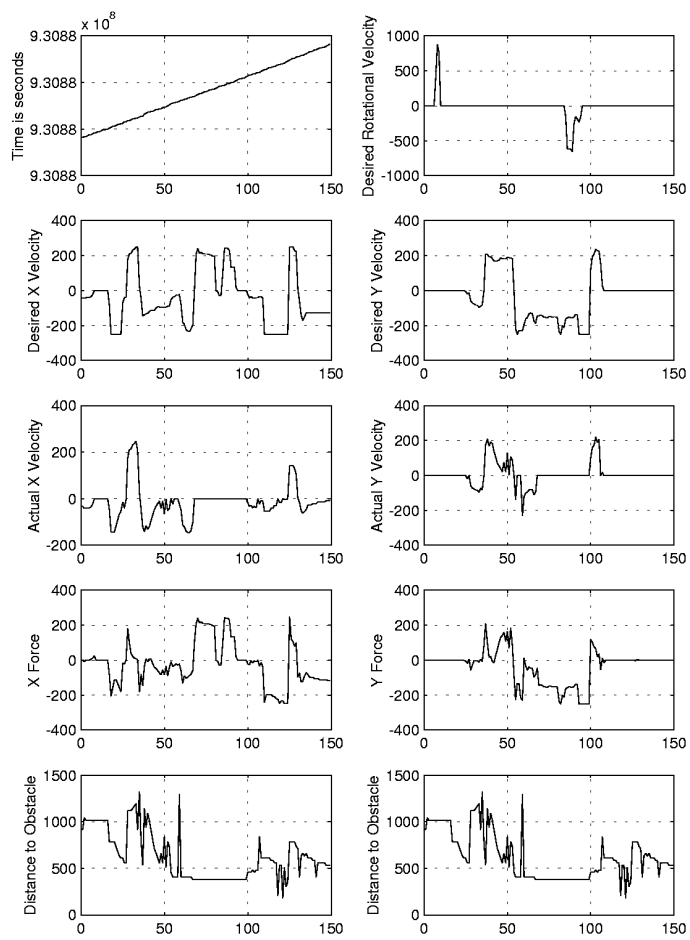

Fig. 5. The behavior of the system during the control from Hong Kong.
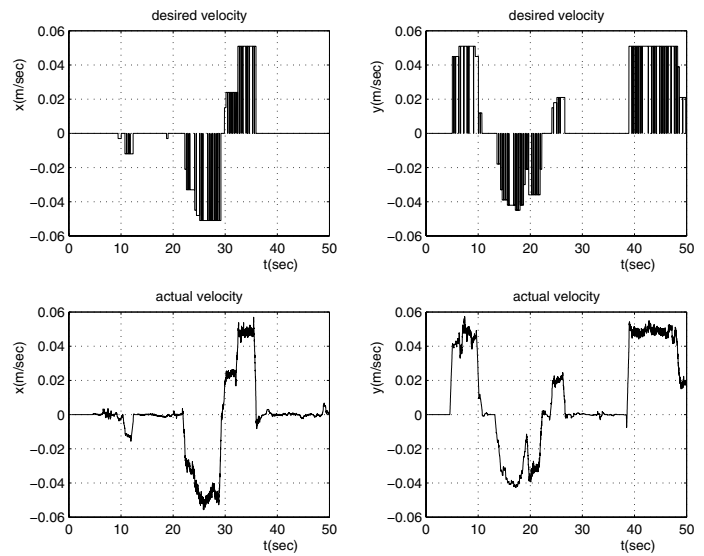

Fig. 6. The velocity performance of the system.

respect to time. The first thing to note is that the desired velocity is a step function since it is a sampled version of the continuous motion of the joystick, where the sampling rate is a function of the time delay faced and the advance 

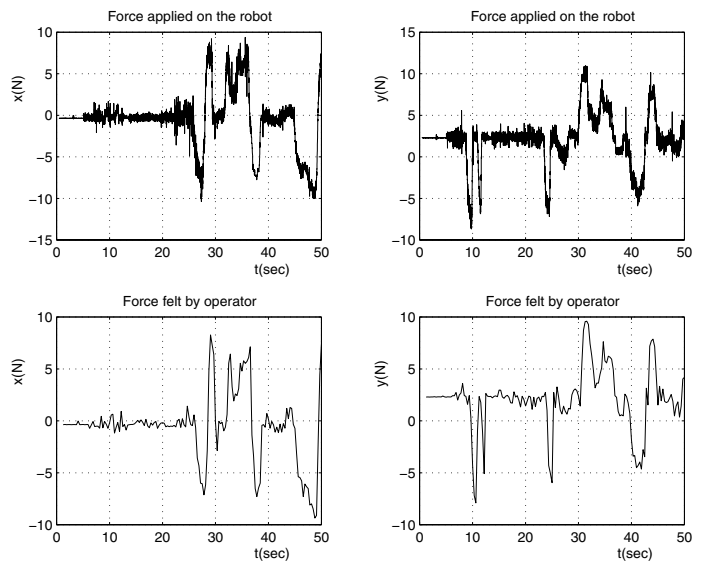

Fig. 7. The force performance of the system.

of the event. More importantly we see that the actually velocity is almost a continuous version of the desired one and it is tracking it very closely.

As for Fig 7 , the first row is the force detected by the force/torque sensor and the second row is the force felt by the operator with respect to time. Note that the sensor is detecting vibrations all the time, this is due to its sensitivity. As for the force felt, it is clear that it is a step function that is almost tracking the actual one. The only slight deformation shown is due to the variable time delay faced between the two machines.

It is clear from these results that the system is stable and event-synchronized since the actual behavior of the robot is tracking the desired one and the force felt by the operator is very close to the actual one the robot is detecting. In addition, the system has high transparency reveled by the fact that the force felt by the operator is very close to the one experienced by the robot. Importantly all those results were achieved under variable time delay with no assumptions regarding it.

The third experiment done was a multi-operator at multi-site mobile manipulator teleoperation with haptic feedback. In this experiment the mobile manipulator (Robotics and Automation lab, Michigan State University), operator1 (Robot Control lab, Chinese University of Hong Kong) and operator2 (Nagoya University, Japan) were connected via the Internet. As seen in the model of the system in Fig 8, the operators send velocity commands and receive haptic information that correspond to the velocity sent by the other operator. This implies that the force felt by each operator corresponds to the intentions of the other one. This setup increases the efficiency of collaboration since it reduces the amount of contradicting commands sent and reflects the intentions of the other operator faster than visual feedback alone.

One of the operators was requested to follow the force felt. This implies that the slave would eventually track the motion of the master. The results of one 


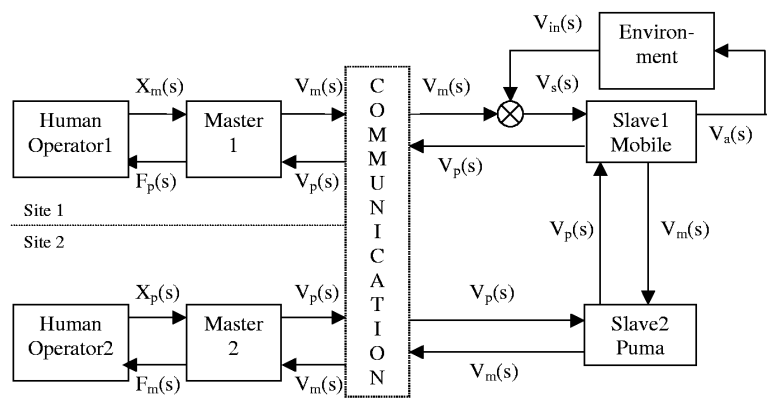

Fig. 8. The system architecture of the multi-operator mobile manipulator teleoperation system implemented.

such experiment are seen in Fig 9, where the operator in Japan is controlling the mobile (slave) and the operator in Hong Kong is operating the puma (master).
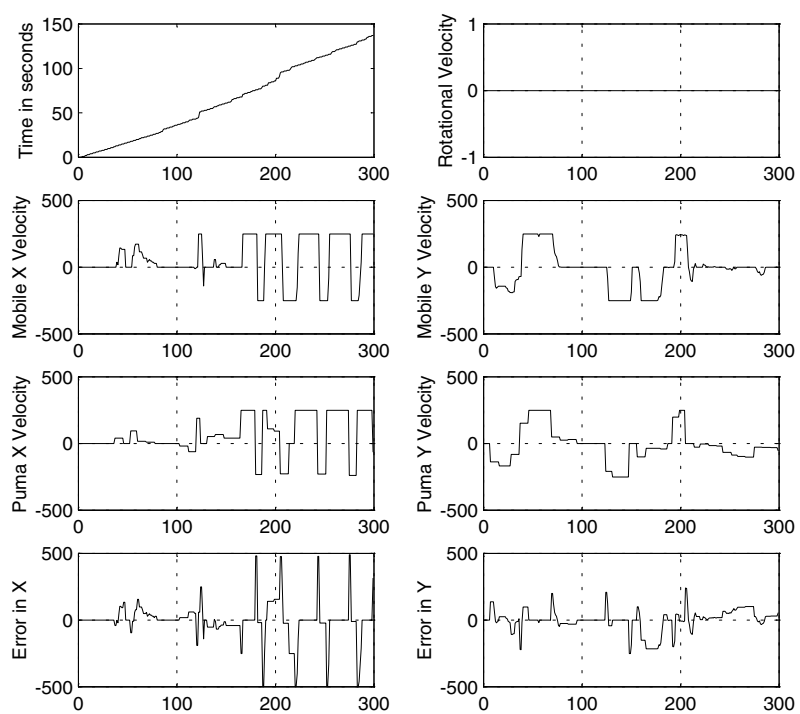

Fig. 9. System Behavior while being controlled from Hong Kong and Japan.

The results show that the desired velocity of the mobile is tracking that of the puma in real-time. The top row of Fig 9 shows a plot of time versus $s$, the event. The other plot in the top row shows the desired rotational velocity. The second row shows the desired velocities of the mobile in $x$ and $y$ directions, $V_{m}$. The Third row plots the desired velocities of the puma in $x$ and $y$ directions, $V_{p}$. The plots of $V_{m}$ and $V_{p}$ also correspond to the forces fed back to operator 2 
and operator1 consecutively. The last row is the error between the mobile and the puma desired velocities in both directions.

The main points to note, are the synchronization and fast response. It is clear that both robots are event synchronized since the shift in direction occurs almost at the same s. Fast response is clear from the sharp decrease in the error between the velocities of the two robots.

Another experiment, that reflects the generality of the approach, is a micro manipulator teleoperation. A PVDF (Polyvinylidence fluoride) micro-tip was used as a piezoelectric sensor for the Internet force-feedback experiment [24]. This tip is about $2.5 \mathrm{~mm}$ long with about $0.8 \mathrm{~mm}$ at the triangular base. The output from this sensor is amplified using an inverted amplifier with feedback gain of 50. Its signal is then feed to a 8255 analog-to-digital conversion (ADC) card connected to a PC for signal transmission to the Internet. This experimental setup is housed in the Advanced Microsystems Laboratory (AML) of The Chinese University of Hong Kong.

Sensor tip is attached to an $\mathrm{x}-\mathrm{y}$ computer-control positioning table, which can be control via the Internet by a force reflection joystick in the Robotics and Automation Laboratory (RAL) at Michigan State University. A cantilever is attached to a vibration drum and has a tip vibration of $100 \mathrm{~mm}$ to $1 \mathrm{~mm}$ from the frequency range of 1 to $120 \mathrm{~Hz}$. The AML sensor tip position can be manipulated by the RAL joystick to contact the vibrating cantilever. The RAL operator observes the AML tip position using a video conferencing software. The force of the vibrating cantilever sensed by the tip is sent to RAL via the Internet. Once the force is received the force feedback joystick plays it. After that the operator generates a new movement command to be sent to the sensor via the Internet.

The experimental results presented here relate to the testing done between Hong Kong and Michigan State. During this experiment the operator (RAL) sends position increment commands and receives force feedback from the sensor (AML). The position increments are sent for both $\mathrm{x}$ and $\mathrm{y}$ axes while the force is sensed only in the y axis. The commands sent are random, which is typical of a teleoperation scenario. This makes approaches based on prediction of forces or virtual forces non-realistic. Therefore, actual force had to be sensed and fed back. Fig 10 presents plots of the force felt by the operator, the force sampled for the sensor and the error between them. As seen the force felt is closely following the one sampled from the sensor. Although this is not occurring at the same time instant, since both plots are with respect to local and not global time, the system is still stable and event synchronized. Despite the random time delay experienced between Hong Kong and Michigan State, the system performance is stable as seen from the error, which is constantly converging to zero and has a small value at all times. 

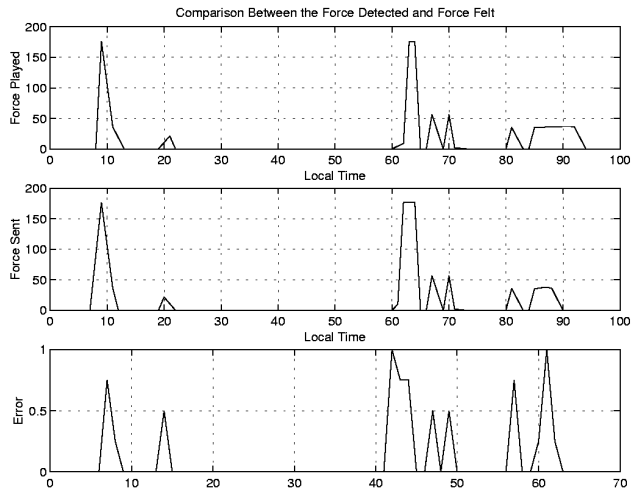

Fig. 10. Comparison between the forces felt and the ones sent.

\section{Conclusions}

This paper examined several issues relating to supermedia in teleoperation over the Internet. The effects of random time delay faced over the Internet were studied. Stability, synchronization and transparency were analyzed and a new planning and control method that ensures those features was presented. The event-based approach was theoretically and experimentally shown to be adequate for the planning and control of teleoperation with haptic feedback.

Several experimental setups were examined and their results presented. All of which showed that the event-based approach resulted in a stable, synchronized and transparent system regardless of time delay and its variance. Other advantages of this approach is that it is independent of the system model, human model and environment model. Therefore, it can be easily adopted to any teleoperation system that includes haptic feedback.

\section{References}

1. D. Kwon, K. Y. Woo, H. S Cho, "Haptic Control of the Master Hand Controller for a Microsurgical Telerobot System", IEEE Int. Conf. on Robotics and Auto., 1999.

2. M. Tanimoto, F. Arai, T. Fukuda, and M. Negoro, "Force Display Method to Improve Safety in Teleoperation System for Intravascular Neurosurgery", IEEE Int. Conf. on Robotics and Automation, Vol. 3, pp. 1728-1733, May 1999.

3. S. E. Everett, R. V. Dubey, "Model-Based Variable Position Mapping for Telerobotic Assistance in a Cylindrical Environment", IEEE Int. Conf. on Robotics and Automation, Vol. 3, pp. 2197-2202, May 1999.

4. L. Hsu, R. Costa, F. Lizarralde, J. Soares, "Passive Arm Based Dynamic Positioning System for Remotely Operated Underwater Vehicles", IEEE Int. Conf. on Robotics and Automation, Vol. 1, pp. 407-412, May 1999. 
5. G. Hirzinger, B. Brunner, J. Dietrich, and J. Heindl, "Sensor-Based Space Robotics-ROTEX and Its Telerobotic Features", IEEE Trans. on Robotics and Automation, Vol. 9, No. 5, October 1993.

6. R. Luo, W. Z. Lee, J. H. Chou, and H. T. Leong, "Tele-Control of Rapid Prototyping Machine Via Internet for Automated Tele-Manufacturing", IEEE Int. Conf. on Robotics and Automation, Vol. 3, pp. 2203-2208, May 1999.

7. R. Anderson, M. Spong, "Asymptotic Stability for Force Reflecting Teleoperators with Time Delay", The Int. Journal of Robotics Research, Vol. 11, April 1992.

8. R. Anderson, M. Spong, "Bilateral Control of Teleoperators with Time Delay", IEEE Trans. on Automatic Control, Vol. 34, No. 5, May 1989.

9. K. Brady, T. J. Tarn, "Internet-Based Remote Teleoperation", Proceedings of the 1998 IEEE Int. Conf. on Robotics and Automation, Leuven, Belgium, May 1998.

10. D. Pai, "ACME, A Telerobotic Measurement Facility for Reality-Based Modelling on the Internet", IROS Workshop on Robots on the Web, Canada, 1998.

11. R. Simmons, "Xavier: An Autonomous Mobile Robot on the Web", IROS Workshop on Robots on the Web, Canada, 1998.

12. N. Y. Chong, T. Kotoku, K. Ohba, K. Komoriya, "Remote Coordinated Controls in Multiple Telerobot Cooperation", IEEE Int. Conf. on Robotics and Auto. 2000.

13. L. F. Penin, K. Matsumoto, S. Wakabayashi, "Force Reflection for Time-delayed Teleoperation of Space Robots", IEEE Int. Conf. on Robotics and Auto., 2000.

14. M. Stein, "Painting on the World Wide Web: The PumaPaint Project", IROS Workshop on Robots on the Web, Canada, 1998.

15. P. Saucy, F. mondada, "KhepOnTheWeb: One Year of Access to a Mobile Robot on the Internet", IROS Workshop on Robots on the Web, Canada, 1998.

16. W. Kim, B. Hannaford, and A. Bejczy, "Force-Reflection and Shared Compliant Control in Operating Telemanipulators with Time Delay", IEEE Trans. on Robotics and Auto., Vol. 8, April 1992.

17. G. Leung, B. Francis, J. Apkarian, "Bilateral Controller for Teleoperators With Time Delay via $\mu$-Synthesis", IEEE Trans. on Robotics and Automation, Vol 11, No. 1, February 1995.

18. M. Otsuka, N. Matsumoto, T. Idogaki, K Kosuge, T. Itoh "Bilateral Telemanipulator System With Communication Time Delay Based on Force-SumDriven Virtual Internal Models", IEEE Int. Conf. on Robotics and Automation, 1995.

19. G. Niemeyer, J. Slotine, "Stable Adaptive Teleoperation", IEEE Journal of Oceanic Engineering, Vol 16, No. 1, January 1991.

20. R. Riedi, M. Course, V. Ribeiro, R. Baraniuk, "A Multifractal Wavelet Model with Application to TCP Network Traffic", IEEE Trans. on Info. Theory April 1999.

21. W. Leland, M. Taqqu, W. Willinger, D. Wilson, "On the Self-Similar Nature of Ethernet Traffic ", IEEE/ACM Trans. on Networking, Vol. 2, No. 1, February 1994.

22. T. J. Tarn, N. Xi, A. Bejczy, "Path-Based Approach to Integrated Planning and Control for Robotic Systems", Automatica, Vol. 32, No. 12, pp. 1675-1687, 1996.

23. I. Elhajj, N. Xi, Y. Liu, "Real-Time Control of Internet Based Teleoperation with Force Reflection", IEEE Int. Conf. on Robotics and Automation, 2000.

24. King W. C. Lai, Carmen K. M. Fung, Wen J. Li, Imad Elhajj, Ning Xi, "Transmission of Multimedia Information on Micro Environment via Internet", International Conference of the IEEE Industrial Electronics Society, Nagoya Japan, 2000. 